ИЗВЕСТИЯ АКАДЕМИИ НАУК ЭСТОНСКОИ ССР. ФИЗИКА * МАТЕМАТИКА PROCEEDINGS OF THE ACADEMY OF SCIENCES OF THE ESTONIAN SSR. PHYSICS * MATHEMATICS

$1987,36,4$

\title{
ГОРЯЧАЯ ЛЮМИНЕСЦЕНЦИЯ МОЛЕКУЛ ХИНИЗАРИНА В СВЕРХЗВУКОВОЙ СТРУЕ И В НИЗКОТЕМПЕРАТУРНОЙ МАТРИЦЕ
}

\author{
(Представил К. К. Ребане)
}

\section{1. Введение}

В последнее время в связи с появлением метода получения свободных охлажденных молекул в сверхзвуковой струе [ $\left.{ }^{1}\right]$ существенно возрос интерес к проблематике колебательной релаксации в возбужденном электронном состоянии больших молекул. Әти процессы отражаются прямым образом в спектре резонансного вторичного свечения (PBC), исследование которого в примесных центрах привело к обнаружению горячей люминесценции (ГЛ) $\left[{ }^{2,3}\right]-$ свечения, которое испускается в ходе колебательной релаксации. Исследование ГЛ позволило выяснить характерные времена и эффективные каналы колебательной релаксации и проводилось в ряде конденсированных систем $\left[{ }^{4}\right]$. Для ароматических молекул в матрицах Шпольского определенные времена распада основных внутримолекулярных колебаний лежат в пикосекундном диапазоне [ $\left.{ }^{5}\right]$. Исследования РВС свободных молекул в струях привели к заключению, что характер релаксационных процессов в этом случае определяется избытком колебательной энергии и условиями возбуждения [ $\left.{ }^{6}\right]$. Для самых низколежащих колебательных состояний (в случае свободных молекул) перераспределения колебательной энергии не происходит и свет испускается из первоначального (резонансного) колебательного состояния. В случае высоколежащих начальных состояний в области, где плотность вибронных состояний высокая, перераспределение (расплывание) колебательной энергии происходит быстро, и времена затухания соответствующих линий лежат в пикосекундном диапазоне. В промежуточных случаях временное поведение более сложное и может наблюдаться кинетика типа квантовых биений $\left[{ }^{7}\right]$. Следует отметить, что наблюдаемая динамика вибронного возбуждения подчиняется известной теореме Фока-Крылова $\left[{ }^{8}\right]$, которая лежит в основе понимания динамической (нетермодинамической) релаксации. Применительно к релаксации примесного центра этот подход был развит в $\left[{ }^{9}\right]$.

В этом контексте возникает фундаментальный вопрос: как идут процессы колебательной релаксации в одной и той же молекуле в разных окружениях или в чем заключается роль матрицы при распаде колебательного возбуждения $\left[{ }^{3,9}\right]$. В настоящей работе проведено сравнение спектров ГЛ молекулы хинизарина, в сверхзвуковой струе аргона $\left[{ }^{10}\right]$ и в замороженном растворе $\boldsymbol{\mu}$-октана. Прямым временным разрешением свечения горячих линий определены соответствующие времена релаксации возбужденных состояний.

Отметим, что работ по прямому временному наблюдению колеба- 
тельной релаксации больших молекул в матрицах пока крайне малло - исследовались молекулы перилена [ $\left.{ }^{11}\right]$, антрацена $\left[{ }^{12}\right]$ и тетрацена [13] в матрицах Шпольского и молекулы нафтазарина в матрицах неона и аргона $\left[{ }^{14}\right]$. Для систем Шпольского получены характерные времена релаксации полносимметричных Франк-Кондоновских колебаний в пределах десятков пикосекунд. Для нафтазарина в неоне зарегистрированы времена, достигающие 320 пс $\left[{ }^{15}\right]$. В $\left[{ }^{16}\right]$ исследовались большие Ван-дер-Ваальсовские комплексы тетрацена в струях (до 200 молекул аргона) и сделан вывод, что время распада внутримолекулярного колебания тетрацена $\left(v=300 \mathrm{~cm}^{-1}\right)$ лежит в наносекундной области. Таким образом, для близких ароматических молекул измеренные времена распада первых, низколежащих колебательных состояний отличаются на несколько порядков в зависимости от окружения. Выявление закономерностей такой зависимости пока еще предстоит.

\section{2. Методика эксперимента}

Хинизарин (1,4-диокси-9,10-антрахинон) очищался перекристаллизацией из раствора $н$-гептана с последующей 110 -кратной зонной плавкой. Растворы хинизарина в н-октане (концентрация $10^{-3}$ моль/л) быстро охлаждались в иммерсионном гелиевом криостате, который откачивался ниже $\lambda$-точки. Спектры возбуждались непрерывным лазером на красителе кумарин-102, накачиваемом ультрафиолетовой линией (413 нм) криптонового лазера. В экспериментах с временным разрешением лазер работал в режиме синхронной накачки и выдавал перестраиваемые от 470 до 530 нм 5 пс импульсы с частотой повторения 80 МГц и средней мощностью 10 мВт. Ширина линии лазера была $3 \mathrm{~cm}^{-1}$. Стационарные спектры хинизарина в матрице Шпольского измерялись системой счета фотонов через монохроматор ДФС-12, спектры свечения свободных молекул в струе регистрировались через монохроматор МДР-2. Установка сверхзвуковой струи описана в [ $\left.{ }^{17}\right]$. Температура камеры сопла (диаметр сопла 150 мкм) составляла 120 $180^{\circ} \mathrm{C}$, давление аргона - 190 торр. Для временных измерений с пикосекундным разрешением использовались спаренный монохроматор с вычитанием дисперсии (спектральное разрешение $6 \mathrm{~cm}^{-1}$ ) [ $\left.{ }^{18}\right]$, электронно-оптическая камера с синхронной разверткой и оптический многоканальный анализатор. Полуширина временной аппаратной функции всей системы 10 пс.

\section{3. Результаты и обсуждение}

Спектры флуоресценции хинизарина в струе $[10,17,19]$ и в матрице $\left[{ }^{20,21}\right]$ обладают богатой вибронной структурой по полносимметричным $\left(A_{1}\right)$ колебаниям. Самые интенсивные линии флуоресценции соответствуют колебаниям с участием квазиароматических циклов, возникающих благодаря внутримолекулярной водородной связи $\left[{ }^{21}\right]$. Соответствующие безразмерные стоксовые потери меньше единицы, т. е. самые большие факторы Франка-Кондона имеют переходы без изменения колебательного состояния (или с изменением соответствующих квантовых чисел на единицу). Ярко выраженные резонансы Ферми (РФ) в спектре возбуждения свободных молекул и сильное перепутывание нормальных координат при электронном переходе свидетельствуют о сильной негармоничности колебаний молекулы.

Нас интересовали в первую очередь нижние колебательные состояния, для которых в свободных молекулах перераспределения энергии 

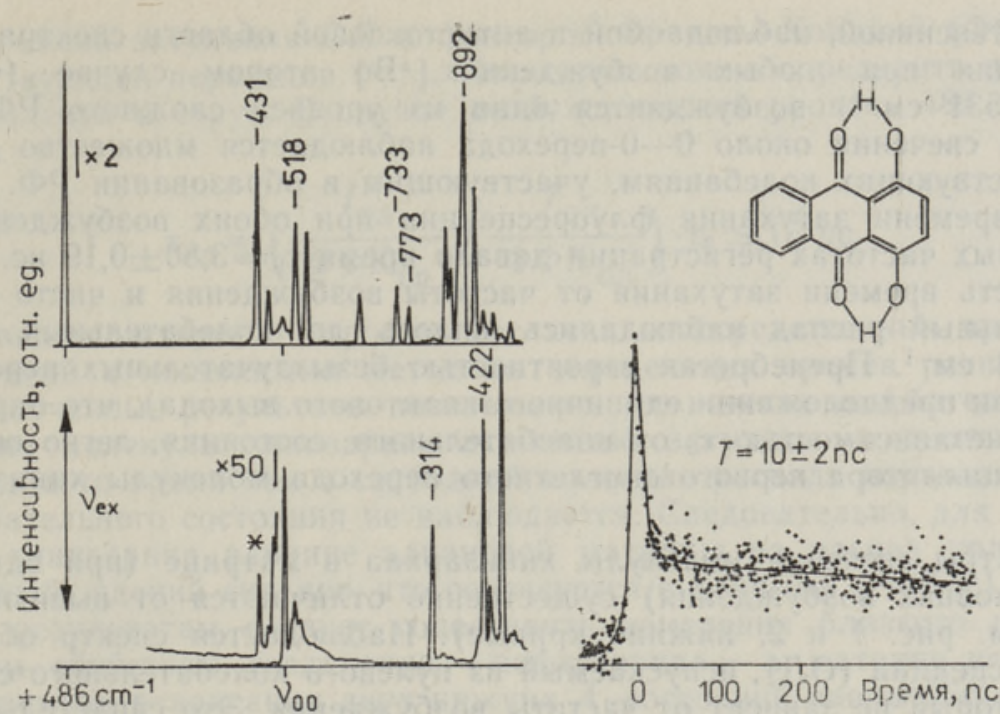

Рис. 1. Слева: спектры свечения молекулы хинизарина в сверхзвуковой струе аргона (сверху) и в матрице н-октана $(T=1,8 \mathrm{~K}$, снизу) при возбуждении на колебательный подуровень $486 \mathrm{~cm}^{-1}$. Цифры над пиками обозначают частоты участвующих в переходе $A_{1}$ колебаний. На нижней части рисунка бесфононная линия сильно реабсорбирована. Справа: временной ход свечения молекулы в матрице на частоте линии горячей люминесценции (в спектре отмечена звездочкой).

не происходит и в спектре флуоресценции проявляются только линии, исходящие из непосредственно возбуждаемого состояния. На верхних кривых рис. 1 и 2 изображены спектры свечения хинизарина в струе аргона при возбуждении на полносимметричные колебательные уровни $v_{1}^{\prime}=486 \mathrm{~cm}^{-1}$ и $v_{2}^{\prime}=531 \mathrm{~cm}^{-1}\left(v_{00}=19917 \mathrm{~cm}^{-1}\right)$. В первом случае в спектре флуоресценции в антистоксовой относительно 0 -0-перехода области появляется несколько сильных линий, из которых самая коротковолновая (переход $\left.S_{1}(v=486) \rightarrow S_{0}(v=431)\right)$ является тақже са-
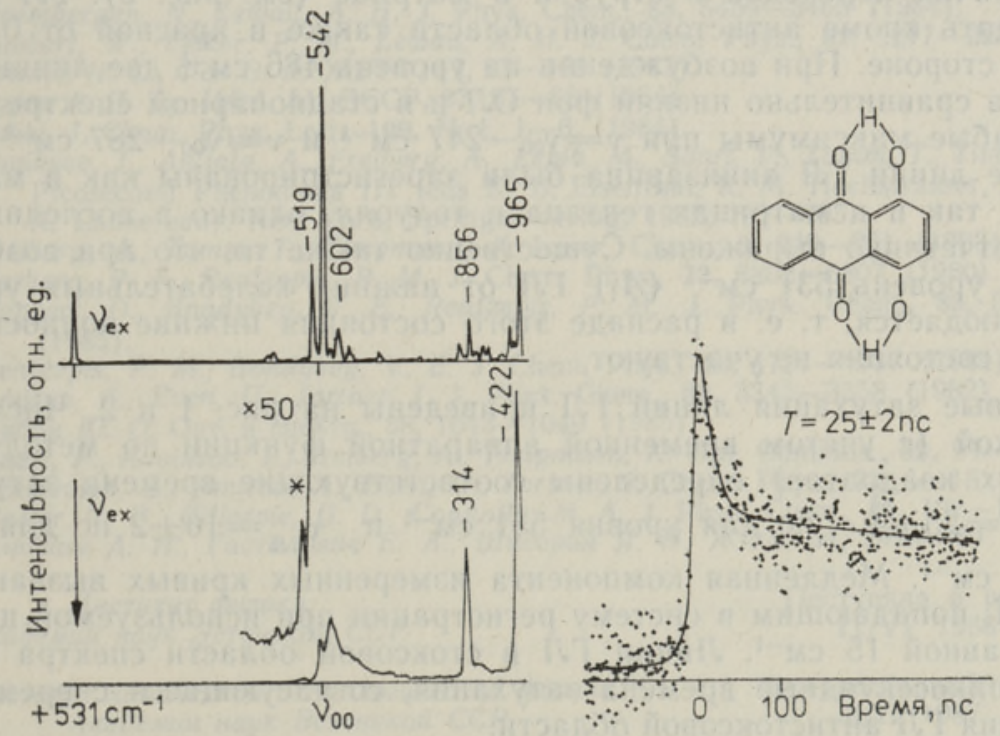

Рис. 2. То же самое, что и на рис. 1. Возбуждение на колебательный подуровень $531 \mathrm{~cm}^{-1}$. 
мой интенсивной, наблюдаемой в антистоксовой области спектра флуоресценции при любых возбуждениях. Во втором случае $\left(v_{\text {возб }}=\right.$ $=v_{00}+531 \mathrm{~cm}^{-1}$ ) возбуждается один из уровней сложного РФ, и в спектре свечения около 0-0-перехода наблюдается множество линий, соответствующих колебаниям, участвующим в образовании РФ. Измерение времени затухания флуоресценции при обоих возбуждениях и на разных частотах регистрации давало время $\tau_{0}=3,80 \pm 0,19$ нс. Независимость времени затухания от частоты возбуждения и чисто экспоненциальный распад наблюдались вплоть до колебательных частот $v \approx 1243 \mathrm{~cm}^{-1}$. Пренебрегая вероятностью безызлучательных переходов (т. е. при предположении единичного квантового выхода), что оправдывается независимостью $\tau_{0}$ от колебательного состояния, легко оценить силу осциллятора первого синглетного перехода молекулы хинизарина $F=1,0$.

Спектры свечения молекулы хинизарина в матрице (при одинаковых условиях возбуждения) существенно отличаются от вышеописанных (см. рис. 1 и 2, нижние кривые). Наблюдается спектр обычной люминесценции (ОЛ), испускаемый из нулевого колебательного состояния, который не зависит от частоты возбуждения. Это свидетельствует о быстром ходе колебательной релаксации в данном случае. Время затухания линий люминесценции $\tau_{0}=3,1 \pm 0,2$ нс. Сокращение времени распада по сравнению с временем распада в свободной молекуле обязано увеличению коэффициента преломления окружения и сдвигу частоты перехода.

Наряду с линиями обычной люминесценции в антистоксовой относительно 0-0-перехода области ( $v_{00}=19113 \mathrm{~cm}^{-1}$ в октане) видны слабые (обратим внимание на изменение масштаба на рис. 1 и 2) линии, которые соответствуют горячим переходам из первоначально возбужденных колебательных подуровней (ГЛ). Основой такой интерпретации служат совпадение частот наблюдаемых линий с частотами, вычисленными по известным колебательным частотам в $S_{0}$ - и $S_{1}$-состояниях, а также спектры возбуждения этих линий. Отметим, что четкий РФ, наблюдаемый в струе и смешивающий состояния на протяжении $15 \mathrm{~cm}^{-1}$, в матрице не наблюдается и это нарушает прямое соответствие горячих переходов в струе и в матрице (см. рис. 2). ГЛ можно наблюдать кроме антистоксовой области также в красной от 0 -0-перехода стороне. При возбуждении на уровень $486 \mathrm{~cm}^{-1}$ две линии попадают на сравнительно низкий фон ОЛ и в стационарном спектре видны как слабые максимумы при $v=v_{00}-247 \mathrm{~cm}^{-1}$ и $v=v_{00}-287 \mathrm{~cm}^{-1}$. Одни и те же линии ГЛ хинизарина были зарегистрированы как в матрице октана, так и в матрицах гептана и толуола, однако в последних они менее отчетливо выражены. Существенно также то, что при возбуждении на уровень $531 \mathrm{~cm}^{-1}\left(A_{1}\right)$ ГЛ от нижних колебательных уровней не наблюдается, т. е. в распаде этого состояния нижние полносимметричные состояния не участвуют.

Кривые затухания линий ГЛ приведены на рис. 1 и 2. Численной подгонкой (с учетом временной аппаратной функции по метолу наименьших квадратов) определены соответствующие времена затухания - $\tau_{\text {гл }}=25 \pm 4$ пс для уровня $531 \mathrm{~cm}^{-1}$ и $\tau_{\text {гл }}=10 \pm 2$ пс для уровня 486 см$^{-1}$. Медленная компонента измеренных кривых вызвана фоном ОЛ, попадающим в систему регистрации при используемой ширине щели равной $15 \mathrm{~cm}^{-1}$. Линии ГЛ в стоксовой области спектра также имели пикосекундные времена затухания, согласующиеся с временами затухания ГЛ антистоксовой области.

Для независимой оценки времени жизни колебательного состояния можно использовать отношение интенсивностей линий ГЛ и ОЛ с уче- 
том временй затухания ОЛ и факторов Франка-Кондона (ФФК) соответствующих переходов $\left[{ }^{2-4}\right]$. Последние можно определить из спектров молекул в струе. В случае возбуждения на уровень $486 \mathrm{~cm}^{-1}$ мы имеем

$$
\tau_{\text {гл }}=\left(\frac{I_{\text {гл }}}{\Phi \Phi \mathrm{K}_{г л}} / \frac{I_{\text {ол }}}{\Phi \Phi \mathrm{K}_{\text {ол }}}\right) \cdot \tau_{0} \simeq 15 \text { пс, }
$$

что свидетельствует о хорошем согласии непосредственных временных измерений с косвенными методами определения времен распада.

Полученные результаты показывают, что нижние колебательные состояния молекулы хинизарина в н-октане затухают за десятки пикосекунд, в то время как в свободной молекуле распада первоначального колебательного состояния не наблюдается. Следовательно, для молекул типа хинизарина влияние алкановой матрицы на распад колебательных возбуждений сильное, что согласуется с данными $\left[{ }^{11,12,13}\right]$. Однако этим результатам следует сопоставить измерения близкого по физическим свойствам соединения - нафтазарина - в матрице неона $\left[{ }^{14}\right]$, где времена релаксации двух нижних $A_{1}$-состояний равны 250 и 320 пс. Столь заметная зависимость времен релаксации родственных соединений требует дальнейшего исследования, в первую очередь измерения времен затухания ГЛ хинизарина в матрицах благородных газов.

Авторы благодарны К. К. Ребане и П. Саари за обсуждение и замечания, а также Э. Рейнот за очистку веществ и изготовление образцов.

\section{Л И Т Е Р А Т У Р А}

1. Levy, D. H. Ann. Rev. Phys. Chem., 31, 197-225 (1980).

2. Ребане К., Саари П. Изв. АН ЭССР. Физ. Матем., 17, № 4, 241-244 (1968).

3. Saari, P., Rebane, K. Solid State Commun., 7, 897-890 (1969).

4. Rebane, K., Saari, P. J. Lumin., 16, 223-243 (1978).

5. Tamm, T., Saari, P. Chem. Phys., 40, № 3, 311-319 (1979).

6. Bloembergen, N., Zewail, A. H. J. Phys. Chem., 88, 5459-5465 (1984).

7. Lambert, W., Felker, P. M., Zewail, A. H. J. Chem. Phys., 81, 2217-2232 (1984).

8. Крылов Н. С., Фок В. А. ЖЭТФ, 17, 93-108 (1947).

9. Пурга А. П. Тр. ИФА АН ЭССР, 27, 57-68 (1964).

10. Subbi, J. Chem. Phys. Lett., 109, № 1, 1-6, (1984).

11. Aaviksoo, J., Anijalg, A., Freiberg, A., Lepik, M., Saari, P., Tamm, T., Timpmann, K. Picosecond Phenomena III (eds K. B. Eisenthal, R. M. Hochstrasser, W. Kaiser, A. Laubereau). New York, Springer-Verlag, 1982, 192-195.

12. Freiberg, A., Tamm, T., Timpmann, K. Laser. Chem., 3, 249-261 (1983).

13. Barbara, P. F., Rentzepis, P. M. J. Chem. Phys., 72, 6802-6807 (1980).

14. Huppert, D., Bondybey, V. E., Rentzepis, P. M. J. Phys. Chem., 89, 5811-5815, (1985).

15. Rentzepis, P. M., Bondybey, V. E. J. Chem. Phys., 80, 4727-4737 (1984).

16. Amirav, A., Even, U., Jortner, J. J. Phys. Chem., 86, 3345-3358 (1982).

17. Субби Ю. О. Опт. и спектр., 58, 1043-1049 (1985).

18. Saari, P., Aaviksoo, J., Freiberg, A., Timpmann, K. Opt. Commun., 39, 94-98 (1981).

19. Smulevich, G., Amirav, A., Even, U., Jortner, J. Chem. Phys., 73, 1-18 (1982).

20. Carter, T. P., Gillespie, G. D., Connolly, M. A. J. Phys. Chem., 86, 192-196 (1982).

21. Аношин А. Н., Гастилович Е. А., Шигорин Я. Н. ЖФХ, 54, 2474-80 (1980).

\section{Институт физики \\ Академии наук Эстонской ССР}

Ннститут химической и биологической физики Академии наук Эстонской ССР
Поступила в редакцию 11/VI 1986

Переработанный вариант 3/II 1987 
T. REINOT, J. SUBBI, J. AAVIKSOO

\section{KINISARIINIMOLEKULI KUUM LUMINESTSENTS OLEHELIKIIRUSLIKUS JOAS JA MADALATEMPERATUURILISES MAATRIKSIS}

On uuritud kinisariinimolekuli võnkerelaksatsiooniprotsesse $n$-oktaani maatriksis ja ülehelikiiruslikus joas. Mölemal juhul on registreeritud kuuma luminestsentsi spektrid; otsese ajalise lahutusega on mõódetud vastavad relaksatsiooniajad ning ära näidatud relaksatsiooniprotsesside olulised erinevused joas ja maatriksis.

\section{T. REINOT, J. SUBBI, J. AAVIKSOO}

\section{HOT LUMINESCENCE OF A QUINIZARINE IN SUPERSONIC JET AND LOW-TEMPERATURE MATRIX}

Vibrational relaxation processes of the quinizarine molecule have been studied in supersonic expansion and in $n$-octane Spol'skii matrix. The hot luminescence spectra have been measured with direct temporal resolution. Lifetimes of 10 and 25 ps have been found for the vibrational levels at 486 and $531 \mathrm{~cm}^{-1}$ in matrix. For the corresponding vibrations in free molecule no relaxation was observed - all emission was hot and decayed with radiational lifetime 3.8 ns. 